\title{
Divergent Co-mordibidites of Zika Virus and Microcephaly in Latin America
}

\author{
Victoria Smerdon ${ }^{\mathrm{a}}$, J. Mike Courage ${ }^{\mathrm{a}}$, Serge Danielson-Francois ${ }^{\mathrm{a}}$
}

\begin{abstract}
Since 2015, a major problem in our society has been the spread of the Zika virus, formally known as ZIKV. ZIKV, originally of African origins, in recent years has spread its way across the Pacific with minor outbreaks before landing in Latin America. The most significant change in ZIKV from discovery is its increasing neurological effects, unique to this disease family. Data has suggested that a pregnant mother having been diagnosed with ZIKV that the child will be subject to congenital disorders as well as Guillain-Barre syndrome. Most notably has been the diagnosis of microcephaly, where a baby is born with an abnormally small head. Some data has suggested a significant increase of rates of microcephaly, but the limitation of small sample sizes and not all populations having been studied prevents a definite conclusion on the effect size of this diagnosis. Therefore, I decided to preform a meta-analysis on existing data sets to determine the likelihood that a child would develop microcephaly, hypothesizing a positive association. My odds ratio did result in a value confirming this confirmation, but because they were statistically insignificant, I had to reject the hypothesis that a pregnant mother's child would develop microcephaly. I presented alternative hypotheses that there is no association because of genomic changes in the virus or not all of Latin American is feeling the effects of microcephaly at the same extent, with Brazil feeling the effects of the virus the strongest, skewing the data and making researchers overestimate the effects of microcephaly. I hypothesized that Brazil has been assumed to be hardest hit because of possible lack of evaluation on past rates of microcephaly, addition of a pesticide to the water, and the Brazilian population being more genetically susceptible to microcephaly. Further research should study genomic changes in the virus strain and testing the Brazilian population original risk of genetic microcephaly.
\end{abstract}

Keywords: Zika virus; Microcephaly; Latin America

\section{Literature Review}

Pre-Epidemic

ZIKV is a flavivirus along with Yellow fever, West Nile virus, and the dengue viruses, characterized with complex life cycles and contain RNA as their genetic core; these characteristics along with urbanization, climate change, and increasing ease of travel can allow for the vector to spread and expand into human populations (Kaslow, 2014). ZIKV was first discovered in 1947 and was spread from mosquitoes in Africa and was commonly misdiagnosed as yellow fever. They identified that the virus has been confined to central and western Africa and to areas of South and Southeast Asia. It remerged in 2007 in Micronesia with symptoms of rashes, conjunctivitis, and arthralgia, but it was on a smaller scale than in Africa and not as deadly. The virus spread through Oceania in 2013, with similar symptoms but the emergence of Guillain-Barre syndrome and fetal microcephaly.

Two different lineages emerged - the first resulting from the initial outbreak in African and the second merging out of the second major outbreak in Southeast Asian. In 2015, patients with similar symptoms of the Southeast Asian lineage were discovered in Brazil (Waggoner, 2016).

ZIKV genomes structurally have two UTRs (5' $-3^{\prime}$ ) and a single long open reading frame encoding a polyprotein, which is split into ten structural and non-structural (NS) proteins, a structure is not very similar to other flaviviruses. There are 25 amino acid substitutions between the pre-epidemic and epidemic strains of the Southeast Asian strain and 50 substitutions with the African strains. They also determined that strains within the same lineage are $89-99 \%$ percent similar, while strains between different ancestries are $85-95 \%$ similar. The NS2A, NS2B, NS4A and NS4B of flaviviruses are small, hydrophobic proteins with incompletely understood functions (Waggoner, 2016).

\section{Current Epidemic}

On February 1, 2016 WHO declared ZIKV a public health emergency of international concern. 76 countries and territories have reported evidence of mosquito-borne ZIKV transmission since 2007 and 59 have been reported after 2015 with 29 having reported microcephaly and other congenital malformations possibly associated with Zika virus connections and 21 having reported Gullain-Barre syndrome (United Nations, 2017).

Brazil is the most affected country in current outbreak with an estimated 500,000-1,500,000 cases since March 2015. It then spread throughout most of South and Central America and was accelerated by El Nino. Travel-related outbreaks were reported in North America, Australia, Europe, and Japan. Transmission easier because majority of cases are places in poverty; poor infrastructure and sanitation allow for the virus to be easily spread as the mosquitoes swarm to sewage, trash, and stagnant water. Overwhelming majority of cases in Brazil were in northeast states of Pernambuco, Bahia, and Paraiba (Baden, 2017).

\section{Symptoms}

ZIKV is transmitted through humans from the Aedes species mosquitoes. It has also been reported to be transmitted through fetal transmission, breast milk, sexual transmission, saliva, and urine, but the significant majority of cases has transmitted from mosquitos (Grischott, 2016). It has also been reported to be transmitted ZIKV in the Americas has had a great upsurge in fetal and pediatric microcephaly throughout Brazil. Various symptoms of ZIKV can be combinations of fever, headache, orbital pain, 
conjunctivitis, rash, myalgias, or arthralgias. Because of lack of access to proper diagnosis methods, patients can be suspected of having DENV or CHIKV disease (Waggoner, 2016).

The most significant presumed effect of the ZIKV breakout has been the significant spread of microcephaly and other congenital malformation in newborns. ZIKV is the first flavivirus to be strong associated with congenital malformations, but first only became obvious in breakouts in Southeast Asia. Microcephaly is a head circumference significantly less than expected for an individual's age and sex, manifested by reduced production of neurons during brain development from a range of mutations in a number of microcephaly genes (Woods, 2005). Prior to the current outbreak, it has been reported from survey data from Yap Island, Federated States of Micronesia, and French Polynesia determined that although all pregnant women were equally susceptible to infection, the baseline risk of microcephaly was determined to be approximately 2 per 10,000 births (Tang, 2016).

Microcephaly was not new to Latin America as it already had a presence prior to the epidemic where there would be about 62 cases of microcephaly a year (European Center for Disease Prevention and Control, 2016) as it is can be caused genetically, where it can be developed as a result of abnormalities in development where there is not the proper production number of neurons and subsequent stages of neuronal development. Microcephaly has been connected to the genes MCPH1, ASPM, CDK5RAP2, CENJPJ, STIL, WDR62, and CEP152, which are responsible for encoding proteins associated with the centrosome, which is responsible for cellar functions such as mitosis. The mutations in these genes can cause mitotic dysfunction, causing these brain abnormalities (Gilmore, 2012). Microcephaly is also associated with different genetic disorders such as Angelman Syndrome, MECP2-related disorder in females, CDKL5-related disorder also in girls, FOXG1-related disorders, among others (Seltzer, 2014).

Microcephaly is one symptom of a wide range of congenital disorders. Other birth defects include brain abnormalities without microcephaly (ex. cerebral atrophy, abnormal cortical formation), neural tube defects and other early brain malformations (ex. ahehcephaly, spina bifida), eye abnormalities (ex. cataract, optic nerve atrophy) and consequences of central nervous system dysfunction (ex. hip displasia, clubfoot). Additionally, Guillan-Barre syndrome is commonly occurring in adults infected with ZIKV. The syndrome is characterized by progressive weaknesses legs and arms and decreased tendon reflexes in the limbs, lasting from two to four weeks (Honein, 2017).

\section{Diagnosis and Treatments}

Currently, ZIKV is difficult to detect because it presents very similar clinical characteristics of the Dengue virus and Chikgungya virus and populations can develop both ZIKV and one of these viruses at the same time. ZIKV can be more accurately diagnoses based on virus isolation, but this can be difficult as they require samples from recovering patients and can still be identified incorrectly, and serological methods, but this requires specialized medical laboratories. More effective techniques being developed include serological detection by immunofluorescence (ELISA) and methods detecting the amount of a genetic nonstructural protein (Hamel, 2016).

ZIKV is currently treated by treating on a symptom basis rather than the disease as a whole as no vaccines are currently available. Research has been done to develop DNA-based vaccine where the vaccines generated antibodies and T-cells in response. Researchers have been working to develop vaccines similar to the Dengue virus vaccine, as they are of similar origins. Additionally, vaccines that will allow the generation of weakened ZIKV strained by altering the NS5 protein to slow the spread of the virus have been researched. Studies have also determine that placental cells may also have a role in resisting ZIKV (Aziz, 2017).

\section{Significance}

Based off of what I have been researching, some data pointed to a positive association between fetal microcephaly and $\mathrm{ZIKV}$, but there was a gap in determining this association as there was no conclusive decision from the scientific community on the extent and how widespread this association occurs in the real world as most studies have been concluded based off of case studies, animal models, small sample sizes, or isolated scenarios, making these results not officially statistically proven. In order to further the development of diagnosis methods and treatments, overall the scientific community needs to expand knowledge about the origins and effects of the virus. Because populations affected by ZIKV are already at risk of microcephaly and other congenital defects and there is limited data available, it in unclear to what extent ZIKV is causing these malformations. My research led me to the question: To what extent is a baby's microcephaly associated with the mother's infection with ZIKV? Most researchers believe there is an association between microcephaly and Zika, but are unable to confirm it because they are lacking statical evidence. Based off of the theories of other researchers, I hypothesized that there is an increased risk of microcephaly for pregnant mothers diagnosed with microcephaly.

This area of research is significant in that determining the risk of microcephaly will firstly determine the likelihood that an infected pregnant mother's baby will develop the condition. Once this has been determined, it provided definite information on the effects of the virus; this information can help fuel the field of researchers on developing diagnosis processes and treatments to effectively combat the current epidemic and hopefully prevent future epidemics from spreading. One of the most significant problems in combatting the virus is the lack of knowledge on its effects and development. Understanding one of the most significant effects allows researchers to successfully fight the virus, slowing the virus in the current epidemic, slowing and treating new epidemics, ultimately preventing new epidemics from arising at this same scale again. 


\section{Methodology}

Based off of the available data, meta-analysis is the best method to examine the relationship between ZIKV and microcephaly. I chose meta-analysis because it allows me to synthesize previous research to determine the real world effects that may have been missed because of limitations such as lack of data, bias, or statical errors. Meta-analysis is systematically reviewing the research examining a particular effect, in this case the pregnant mother infected with ZIKV on microcephaly, and combining the results of independent studies to estimate the population effect size. Because of the limitations of previous studies, a meta-analysis would comprehensively review the real-world association between ZIKV and microcephaly. Meta-analysis will increase the statistical power of research already been done, making for a more precise estimate of the effect size of microcephaly. Once the effect size has been determined, this will allow for further research to be completed with a more significant understanding of the virus, allowing for more effective vaccines and diagnostics.

I carried out my meta-analysis by searching for articles in the University of Michigan Dearborn Mardigian library database. I did not limit articles based off of language, but I limited them based off of year, 2015 to the present, location, South America and the Caribbean, and the studies had to have reported results that could be compared in meta-analysis. I also limited my studies to real-world examples of people contracting ZIKV rather than laboratory studies of animals and experimental models. I prevented against publication bias by looking for unpublished articles at arxiv.org, but found nothing relevant to my question. I used software from meta-analysis.com, Comparative Meta-Analysis, to carry out the statistical tests. I inserted information on case-control studies or p-values to determine the odds ratio, log odds ratio, and the standard error. Once this information is synthesized together by the software, it will report an odds ratio, 95\% confidence interval, p-values, and $\mathrm{Z}$ score.

After excising irrelevant studies, studies that do not follow my defined requirements, and incomparable studies, I was only able to compare five studies. I used the following articles: "Congenital Zika virus syndrome in Brazil: a case series of the first 1501 livebirths with complete investigation," "Association between Zika virus infection and microcephaly in Brazil, January to May, 2016: preliminary report of a case-control study," Zika Virus Infection in Pregnant Women in Rio de Janeiro," "Increase in Reported Prevalence of Microcephaly in Infants Born to Women Living in Areas with Confirmed Zika Virus Transmission During the First Trimester of Pregnancy - Brazil, 2015," "Birth Defects Among Fetuses and Infants of US Women With Evidence of Possible Zika Virus Infection During Pregnancy," "Estimating the Number of Pregnant Women Infected With Zika Virus and Expected Infants With Microcephaly Following the Zika Virus Outbreak in Puerto Rico, 2016." These sources all followed my coding as they were all studying Latin America, looking at real populations, and were comparable through the metaanalysis software. The article author, data, and weighted means are shown in the chart below as well as the resulting odds ratio, $\log$ ratio, and standard error.

\begin{tabular}{|c|c|c|c|c|c|c|c|}
\hline Author & $p$ value & Tails & Sample size & $\begin{array}{l}\text { Effect } \\
\text { direction }\end{array}$ & Odds ratio & $\begin{array}{l}\text { Log odds } \\
\text { ratio }\end{array}$ & $\begin{array}{l}\text { Standard } \\
\text { Error }\end{array}$ \\
\hline Franca et al & 0.070 & 1 & 602 & Positive & 1.245 & 0.219 & 0.148 \\
\hline Authors & $\begin{array}{l}\text { Microcephal } \\
\text { y Case }\end{array}$ & $\begin{array}{l}\text { Pregnant } \\
\text { with Zika }\end{array}$ & $\begin{array}{l}\text { Microcephal } \\
\text { y Control }\end{array}$ & $\begin{array}{l}\text { Pregnant } \\
\text { Control }\end{array}$ & Odds ratio & $\begin{array}{l}\text { Log odds } \\
\text { ratio }\end{array}$ & $\begin{array}{l}\text { Standard } \\
\text { Error }\end{array}$ \\
\hline $\begin{array}{l}\text { Elllington et } \\
\text { al }\end{array}$ & 180 & 7800 & 12 & 190 & 0.365 & -1.007 & 0.307 \\
\hline Honein et al & 8 & 167 & 14 & 271 & 0.927 & -0.075 & 0.454 \\
\hline Araujo et al & 21 & 21 & 7 & 73 & 10.459 & 2.345 & 0.502 \\
\hline
\end{tabular}




\begin{tabular}{|l|l|l|l|l|l|l|l|}
\hline Author & p value & Tails & Sample size & $\begin{array}{l}\text { Effect } \\
\text { direction }\end{array}$ & Odds ratio & $\begin{array}{l}\text { Log } \\
\text { ratio }\end{array}$ & $\begin{array}{l}\text { odds } \\
\text { Error }\end{array}$ \\
\hline Brasil et al & 58 & 134 & 7 & 73 & 4,541 & 1.507 & 0.426 \\
\hline
\end{tabular}

The meta-analysis will be carried out through the combination of observations in different studies to draw conclusions on the real world effects to determine the weighed mean effect size and confidence interval. Then, the effect size is normalized into a $\mathrm{Z}$ score to determine statistical significance in the real world, which could then be translated in the p value. The odds ratio showing how likely that a baby whose mother contracted ZIKV will develop microcephaly will be determined from this information (Hunt, 1999).

\section{Results}

Meta-analyses from the medical discipline typically aim to produce an odds ratio which determines the likelihood that a baby will develop microcephaly, a $\mathrm{Z}$ value which indicates how many possible standard deviations an element can be away from the mean, and a $\mathrm{P}$ value which determines the significance of my results by determining the probability that the odds ratio is accurate. I chose to report my results in a random effects model. I chose this model because my analysis is the result of accumulating data from a series of studies that had been performed by researchers operating independently. They are all looking at populations across Latin America, are reporting from across the world, and report their data as different variables, therefore cannot be considered identical and studied under the fixed effects model.

The meta-analysis program returned under random effects and odds ratio of 1.727 with a $95 \%$ confidence interval (0.6694.458). Since the value is greater than one, this mean that babies whose mothers developed microcephaly at birth are at risk for microcephaly. The $\mathrm{Z}$ value is 1.1259 , meaning that the the possible standard deviations is 1.129 . Despite the odds ratio being greater than one, the $\mathrm{p}$ value is 0.259 ; because the $\mathrm{p}$-value is greater than 0.05 , I have to reject the hypothesis that microcephaly is associated with a mother developing ZIKV during pregnancy because my results are no longer statistically significant, so the association presented in my odds ratio is no longer definitive.

\begin{tabular}{|l|l|l|l|l|}
\hline Odds ratio & Lower limit & Upper limit & Z value & P value \\
\hline 1.727 & 0.669 & 4.458 & 1.129 & 0.259 \\
\hline
\end{tabular}

\section{Discussion}

Alternative Hypotheses

The meta-analysis showing no correlation between ZIKV and microcephaly provides a stark contrast to a different conclusion made by researchers on the epidemic in French Polynesia. Research was done on the association between ZIKV and microcephaly in French Indonesia from 2013-2015 to determine the potential risk for the Latin American epidemic. This research reported that mothers infected with ZIKV are at an increase risk of $1 \%$ of their baby developing microcephaly (Thiet, 2016) Although this is a small increased risk, this still points to the positive association that my results did not suggest.

My data analysis may have shown different presumed risk of microcephaly firstly may imply that there are genomic differences between the Southeast Asian strain and the Latin American strain that have changed how ZIKV development microcephaly. Genomic analyses of the ZIKV strains have shown differences in Southeast Asian strain and the Latin American strain. Researchers reported that the Latin American strains are fairly similar, but some differences remain in the "amino acid residues external exposed...[of] South American ZIKV" (Kochakarn, 2016). Because of these differences in the genome of the virus, this may have accounted for the change of the prevalence of microcephaly.

An additional alternate hypothesis may be that although researchers have reported that ZIKV has been causing fetal microcephaly in all of Latin America, but my resulted may have implied that this is a regional problem because of Brazil. Researchers have reported less of a prevalence of microcephaly among Colombian populations, while still under the same risk of microcephaly. Colombia, although having experience the second highest number of cases of Zika virus in South America, did not experience a peak in congenital birth defects as reported in other areas of Latin America, but did experience a significant increase of Guillain-Barre syndrome (Machado-Alba, 2016). This difference between Colombia and the presumed increase of microcephaly in Brazil may account for the rejection of my hypothesis.

The Brazilian Problem

The genomic changes of the South American virus and the inconsistency of microcephaly occurrences throughout Latin America may account for the rejection of my hypothesis that microcephaly is associated with maternal ZIKV in Latin America. One possible claim on why researchers in Brazil have been reporting the severe increase in microcephaly is that this increased 
risk may be of a negligible difference because prior to the ZIKV epidemic, Brazil has already been at risk of microcephaly, so researcher's analysis of the risk may have shown negligible increases in risk of microcephaly because it did not take into account the prior risk to microcephaly.

Furthermore, researchers have theorized that these birth defects could be because a new chemical, larvicide, was being used as a pesticide in Brazil's water supply beginning in 2015 to prevent the spread of mosquitoes. Research done on zebrafish has shown that this chemical is not the cause of microcephaly; although it is lethal in large doses, it does not cause any brain malformations (Dzieciolowska, 2017) This pesticide despite this conclusion on zebra fish may still be a possible reason on why the microcephaly problem is occurring most significantly in Brazil.

Therefore, this incorrectly reported increased association may be because the Brazilian population possibly being genetically more susceptible to microcephaly and other congenital birth defects. As microcephaly has been previously identified as genetic and was already present in the Brazilian population, this is one of many plausible solutions on why the Brazilian populations have been reported to be significantly affected by microcephaly while other Latin American nations did not.

Further Research

More research can help solve many of these uncertainties. Further research should be focus on studying why the Brazilian population has been greatly affected by microcephaly through specifically genetically tests. Additional research should be focused on definitively determining which populations are at risk for microcephaly because of ZIKV and to what extent they are at risk. Research also should be done additionally at the genomic level to determine how the virus has been changing on the genomic level and how this will be affecting the risk of microcephaly or other congenital malformations as the regions of the genome changing in the strain's role in its development is unknown. Once these uncertainties are solved through accurate and significant studies researches can focus their work on working toward efficient and effective forms of diagnosing ZIKV and creating vaccines.

\section{Limitations}

Firstly, my paper is limited based off of the previous work of the scientific community. As the ZIKV and microcephaly have only become a significant problem in the last few years, there is a lack of articles and research done to study the virus. The scientific community is currently attempting to thoroughly research the virus and its effects, but there are only so many comparable articles for a meta-analysis. Additionally, because many of the infected populations are living in poorer and more isolated areas of Latin America, there is in general a lack of comprehensive data. Not all populations infected are thoroughly researched; there may be differences in the prevalence of the disease and microcephaly, especially in Brazil.

Due to these reasons, my results could have been skewed or inaccurate. Furthermore, how I coded my articles severely limited the number of articles I could synthesize in my meta-analysis. In the meta-analysis program only similar trials can be synthesized together, therefore many of the articles I chose had to be excised because of that. Despite the fact I attempted to prevent against language and publication bias, the articles I compared were not perfectly heterogenous, putting a focus on Brazil as many studies completed are focused on Brazil. Additionally, I was subject to publication bias due to the fact that I was only able to find published studies as studies without significant results are unlikely to be published; this bias may results in either a trivial or substantial bias in my paper, depending on how many studies went unpublished.

Furthermore, meta-analysis has its limitations. Meta-analysis can only be successful with good trials being compared; because many of the articles I used may have also had limited analyses, it could have skewed my data. Future researchers should look to studies that are able to return the most significant, comprehensive, and unbiased results. Meta-analysis is the best way based on available, public data to determine the effect size of the development of microcephaly, but more research is needed to further examine this problem.

\section{Conclusion}

In the recent ZIKV epidemic, the most significant problem has been the diagnosis of microcephaly, where a baby is born with an abnormally small head. Some data has suggested a significant increase of rates of microcephaly, but the limitation of small sample sizes and not all populations having been studied prevents a definite conclusion on the effect size of this diagnosis. Therefore, I decided to preform a meta-analysis on existing data sets to determine the likelihood that a child would develop microcephaly, hypothesizing a positive association. I focus my analysis on Latin American nations and statistics on real populations of affect patients. My odds ratio did result in a value confirming this confirmation, but because they were statistically insignificant, I had to reject the hypothesis that a pregnant mother's child would develop microcephaly. I theorized alternative hypotheses that there is no association because of the genomic evolution in the virus or not all of Latin American is feeling the effects of microcephaly similarly, with Brazil feeling the effects of the virus the strongest, skewing the data and making researchers overestimate the effects of microcephaly. I hypothesized that Brazil has been assumed to be hardest hit because of lack of information on past rates of microcephaly in ZIKV, possibly addition of a pesticide to the water, and the Brazilian possibly population being more genetically susceptible to microcephaly. My analysis was limited based off of how I coded my articles, lack of data in the scientific community, and my potential bias in the excising of my articles. Ultimately, ZIKV is a major problem challenging scientific researchers. Because of lack of knowledge on how the virus has been changing and the full extent of its effects, determining the risk of microcephaly is not an easy problem to solve, but it is necessary in order to help develop diagnosis methods, treatments, and vaccines in order to help subside the current epidemic and prevent or subdue other epidemics around the world. This problem can only be combatted with the full cooperation and effort of scientific communities to obtain data and analyze it to effectively understand the virus and advance its diagnosis process and treatment. 


\section{References}

Aziz, Hafsa, Aadarash Zia, Amania Anwer, Muneeba Aziz, Shazia Fatima, and Muhammad Faheem. Zika virus: Global health challenge, threat and current situation. Journal of Medical Virology 89, no. 6 (2017): 943-51. doi:10.1002/jmv.24731.

Baden, Lindsey R., et al. Zika Virus. The New England journal of Medicine 374.16 (2016): 1552-63. ProQuest. Web. 6 Jan. 2017

Dzieciolowska, Stefania, Anne-Laure Larroque, Elizabeth-Ann Kranjec, Pierre Drapeau, and Eric Samarut. "The larvicide pyriproxyfen blamed during the Zika virus outbreak does not cause microcephaly in zebrafish embryos." Scientific Reports 7 (2017): 40067. doi:10.1038/srep40067

Ellington, Sascha R., Owen Devine, Jeanne Bertolli, Alma Martinez Quiñones, Carrie K. Shapiro-Mendoza, Janice Perez-Padilla, Brenda Rivera-Garcia, Regina M. Simeone, Denise J. Jamieson, Miguel Valencia-Prado, Suzanne M. Gilboa, Margaret A. Honein, and Michael A. Johansson. Estimating the Number of Pregnant Women Infected With Zika Virus and Expected Infants With Microcephaly Following the Zika Virus Outbreak in Puerto Rico, 2016. JAMA Pediatrics 170, no. 10 (2016): 940. doi:10.1001/jamapediatrics.2016.2974.

European Center for Disease Prevention and Control. Rapid Risk Assessment: Zika Virus Epidemic in the Americas: Potential Association with Microcephaly and Guillain Barre Syndrome. (2016)

Gilmore, Edward C., and Christopher A. Walsh. Genetic causes of microcephaly and lessons for neuronal development. Wiley Interdisciplinary Reviews: Developmental Biology 2, no. 4 (2012): 461-78. doi:10.1002/wdev.89.

Grischott, Franca, Milo Puhan, Christoph Hatz, and Patricia Schlagenhauf. Non-vector-borne transmission of Zika virus: A systematic review. Travel Medicine and Infectious Disease 14, no. 4 (2016): 313-30. doi:10.1016/j.tmaid.2016.07.002.

Hamel, Rodolphe, Florian Liégeois, Sineewanlaya Wichit, Julien Pompon, Fodé Diop, Loïc Talignani, Frédéric Thomas, Philippe Desprès, Hans Yssel, and Dorothée Missé. Zika virus: epidemiology, clinical features and host-virus interactions. Microbes and Infection 18, no. 7-8 (2016): 441-49. doi:10.1016/j.micinf.2016.03.009.

Hamer, Davidson. Faculty of 1000 evaluation for Congenital Zika virus syndrome in Brazil: a case series of the first 1501 livebirths with complete investigation. F1000 - Post-publication peer review of the biomedical literature, 2016. doi:10.3410/f.726480017.793521176.

Honein, Margaret A., April L. Dawson, Emily E. Petersen, Abbey M. Jones, Ellen H. Lee, Mahsa M. Yazdy, Nina Ahmad, Jennifer Macdonald, Nicole Evert, Andrea Bingham, Sascha R. Ellington, Carrie K. Shapiro-Mendoza, Titilope Oduyebo, Anne D. Fine, Catherine M. Brown, Jamie N. Sommer, Jyoti Gupta, Philip Cavicchia, Sally Slavinski, Jennifer L. White, S. Michele Owen, Lyle R. Petersen, Coleen Boyle, Dana Meaney-Delman, and Denise J. Jamieson. Birth Defects Among Fetuses and Infants of US Women With Evidence of Possible Zika Virus Infection During Pregnancy. Jama 317, no. 1 (2017): 59. doi:10.1001/jama.2016.19006.

Hunt, Morton M. How science takes stock: the story of meta-analysis. New York: Russell Sage Foundation, 1999.

Interim Guidelines for the Evaluation of Infants Born to Mothers Infected with West Nile Virus During Pregnancy. Morbidity and Mortality Weekly Report 53, no. 7 (2004): 154-57. http://www.jstor.org/stable/23315341

Kaslow, Richard A., Lawrence R. Stanberry, and Le Duc James W. Viral infections of humans: epidemiology and control. New York: Springer, 2014.

Kochakarn, Theerarat, Namfon Kotanan, Krittikorn Kümpornsin, Duangkamon Loesbanluechai, Monta Thammasatta, Prasert Auewarakul, Prapon Wilairat, and Thanat Chookajorn. Comparative genome analysis between Southeast Asian and South American Zika viruses.Asian Pacific Journal of Tropical Medicine 9, no. 11 (2016): 1048-054. doi:10.1016/j.apjtm.2016.10.002.

Machado-Alba, Jorge Enrique, Manuel E. Machado-Duque, Andrés Gaviria-Mendoza, and Viviana Orozco-Giraldo. Diagnosis of neurological disorders and the Zika virus epidemic in Colombia 2014 -2016. International Journal of Infectious Diseases 51 (2016): 133-34. doi:10.1016/j.ijid.2016.09.010.

Oliveira, Wanderson Kleber De, Juan Cortez-Escalante, Wanessa Tenório Gonçalves Holanda De Oliveira, Greice Madeleine Ikeda Do Carmo, Cláudio Maierovitch Pessanha Henriques, Giovanini Evelim Coelho, and Giovanny Vinícius Araújo De França. "ncrease in Reported Prevalence of Microcephaly in Infants Born to Women Living in Areas with Confirmed Zika Virus Transmission During the First Trimester of Pregnancy — Brazil, 2015. MMWR. Morbidity and Mortality Weekly Report 65, no. 9 (2016). doi:10.15585/mmwr.mm6509e2er.

Seltzer, Laurie E., and Alex R. Paciorkowski. Genetic disorders associated with postnatal microcephaly. American Journal of Medical Genetics Part C: Seminars in Medical Genetics 166, no. 2 (2014): 140-55. doi:10.1002/ajmg.c.31400.

Tang, Bor Luen. Zika virus as a causative agent for primary microencephaly: the evidence so far. Archives of Microbiology 198, no. 7 (2016): 595-601. doi:10.1007/s00203-016-1268-7.

Thiet, Mari-Paule. Faculty of 1000 evaluation for Association between Zika virus and microcephaly in French Polynesia, $2013-$ 15: a retrospective study. F1000 - Post- publication peer review of the biomedical literature, 2016. doi:10.3410/f.726226588.793521422.

United Nations. World Health Organization. Situation Report: Zika Virus, Microcephaly,Guillain-Barre Syndrome. 2017.

Waggoner JJ, Pinsky BA. 2016. Zika virus: diagnostics for an emerging pandemic threat. J Clin Microbiol 54:860-867. doi:10.1128/JCM.00279-16.

Woods, C. Geoffrey, Jacquelyn Bond, and Wolfgang Enard. Autosomal Recessive Primary Microcephaly (MCPH): A Review of Clinical, Molecular, and Evolutionary Findings. The American Journal of Human Genetics 76, no. 5 (2005): 717-28. doi:10.1086/429930.

Zika virus infection in pregnant women in Rio de Janeiro. Current Medicine Research and Practice 6, no. 2 (2016): 95. doi:10.1016/j.cmrp.2016.03.003. 